A) Check for updates

Cite this: Food Funct., 2022, 13, 574

\section{Evaluation of novel nutraceuticals based on the combination of oat beta-glucans and a green coffee phenolic extract to combat obesity and its comorbidities. A randomized, dose-response, parallel trial}

\author{
Raquel Mateos, (D) Joaquín García-Cordero, Laura Bravo-Clemente (D) and \\ Beatriz Sarriá (D) *
}

\begin{abstract}
Obesity and its associated comorbidities are a major public health concern worldwide. Reduced energy intake and increased physical activity interventions have limited success in the long term. Nutraceuticals might be an alternative means to help lose weight and reduce obesity-associated cardiometabolic risk factors without changes in the habitual diet. The objective of the present study was to comparatively evaluate the efficiency of nutraceuticals based on the combination of a decaffeinated green coffee bean extract (GCBE) and two types of oat beta-glucans (BG) with different physiochemical properties on obesity related biomarkers in overweight/obese subjects. A randomized, dose-response, parallel, blind study was carried out in four groups of subjects ( $n=15$ each) who consumed for 6 weeks, twice a day, a nutraceutical containing $3 \mathrm{~g} \mathrm{~d}^{-1}$ or $5 \mathrm{~g} \mathrm{~d}^{-1}$ doses of $35 \%$ or $70 \% \mathrm{BG}$ and a fixed amount of GCBE providing $600 \mathrm{mg} \mathrm{d}^{-1}$ of phenols. 35\% BG showed a 10 and 100 times higher molecular weight and viscosity, respectively, compared to $70 \%$ BG. Food intake, anthropometry and different cardiometabolic markers were assessed at the beginning and end of the intervention. According to the general model of variance with repeated measure analysis, the intervention caused positive changes in the levels of total cholesterol, LDL cholesterol, VLDL cholesterol, triglycerides, alanine aminotransferase, aspartate aminotransferase, haemoglobin A1c, insulin, systolic blood pressure (SBP), total body fat percentage (TBF\%), visceral fat percentage, and waist and hip circumferences without differences among the treatments, except for SBP and TBF\%. Looking into the rates of change [(end value - beginning value)/beginning value] of these parameters, $5 \mathrm{~g}-70 \%$ BG was the treatment that lowered TBF\% the most. In conclusion, $5 \mathrm{~g}-70 \% \mathrm{BG}$ may be more effective in helping to lose weight and additionally, it produced the least bloating according to participants' subjective perception.
\end{abstract}

Received 14th July 2021, Accepted 27th November 2021

DOI: $10.1039 /$ d1fo02272e

rsc.li/food-function sidering the difficulties for patients in adopting long-term changes in dietary habits and increasing physical activity.

Nutraceuticals or dietary supplements have proved to be important tools to fight against various pathologies, including overweight/obesity, thanks to the high content of bioactive compounds with health beneficial effects. Recently, the scientific community has focused its attention on nutraceuticals with high soluble dietary fibre (SDF) content or extracts from plants rich in phenolic compounds (PC). Among SDF outstand $\beta$-glucans (BG), which may form viscous solutions at low concentration and are highly fermentable in the colon. ${ }^{3}$ BG have been associated with numerous positive health effects, which have been recently reviewed in a study by García-Cordero et $a .^{4}$ Amounting evidence on the health beneficial effects of BG led the European Food Safety Authority (EFSA) to issue favourable opinions regarding the consumption of $\mathrm{BG}$, stating that
Department of Metabolism and Nutrition, Institute of Food Science, Technology and Nutrition (ICTAN-CSIC), Spanish National Research Council (CSIC), José Antonio Nováis 10, 28040 Madrid, Spain. E-mail: beasarria@ictan.csic.es; Tel: +34915492300 
regular consumption of at least $3 \mathrm{~g} \mathrm{~d}^{-1}$ of BG contributes to the maintenance of normal blood cholesterol levels, ${ }^{5}$ with a specific effect of oat BG reducing blood cholesterol concentration. ${ }^{6}$ In addition, BG also show hypoglycaemic effects, ${ }^{7}$ which have resulted in another favourable opinion by the EFSA establishing the consumption of $4 \mathrm{~g}$ of BG for every $30 \mathrm{~g}$ of digestible carbohydrates for reducing post-prandial glycaemic responses. ${ }^{8}$ Moreover, BG promote satiety by modulating the secretion of orexigenic and anorexigenic gastrointestinal hormones, resulting in lower caloric intake, contributing to their hypoglycaemic and hypolipemic effects. ${ }^{9,10}$ As a prebiotic, BG may increase the proliferation of Bacteroidetes, Bacteroides and Prevotella and decrease Firmicutes and Dorea, which have been correlated with a decrease in body mass index (BMI), waist circumference, blood levels of triglycerides (TG), low density lipoprotein cholesterol (LDL-C), high density lipoprotein cholesterol (HDL-C) and glucose, and blood pressure. ${ }^{11}$

On the other hand, phenolic compounds have extensive health beneficial properties. ${ }^{12}$ Among PC, hydroxycinnamic acids outstand due to their high consumption and relevant health beneficial properties. ${ }^{4}$ Briefly, hydroxycinnamates may improve cardiovascular health by decreasing total cholesterol (T-C), LDL-C and TG levels and improving the blood pressure and plasma antioxidant capacity in subjects with hypercholesterolemia ${ }^{13}$ and high blood viscosity. ${ }^{14}$ These effects have been related to the inhibition of key enzymes involved in fatty acid synthesis, improved sensitivity to insulin and leptin, reduced expression of genes involved in lipogenesis, and increased lipid $\beta$-oxidation in the liver. ${ }^{15}$ In addition, hydroxycinnamates may also improve glycaemic homeostasis and insulin sensitivity, as shown in both pre-diabetic ${ }^{16}$ and type 2 diabetic patients, ${ }^{17}$ by different mechanisms such as reducing glucose uptake in the gastrointestinal tract, ${ }^{24}$ increasing glucose uptake in muscle and adipose tissue, enhancing the expression of glucose transporter GLUT4 $4^{19,25}$ and reducing the activity of glucose 6-phosphatase and phosphoenolpyruvate carboxykinase in the liver. ${ }^{15,18,19}$ Furthermore, hydroxycinnamates have shown to affect satiety and delay gastric emptying, ${ }^{20}$ affecting also the intestinal microbiota and increasing the metabolic activity and count of Bifidobacterium spp. ${ }^{21}$ The main dietary source of hydroxycinnamates in Western societies is coffee, and unroasted, green coffee beans are especially rich in these phenolic compounds. Supplements containing green coffee bean extract have shown promising effects against obesity. $^{22-25}$

Bearing this in mind, the combination of BG and hydroxycinnamates might be a promising nutritional tool to fight against obesity and its associated pathologies. As far as we know, there are no commercial nutraceuticals that consist of the combination of BG and hydroxycinnamates. Therefore, the objective of the present study was to comparatively investigate the effects of the regular consumption of novel nutraceuticals consisting of the combination of two types of BG (35\% or $70 \%$ $\mathrm{BG})$ at two doses ( 3 or $5 \mathrm{~g} \mathrm{~d}^{-1}$ ) and hydroxycinnamates from green coffee bean extract on overweight/obesity and its associated pathologies.

\section{Experimental}

\subsection{Test compounds and nutraceuticals}

Beta-glucans. PromOat ${ }^{\circledR}$ Beta Glucan with $35 \%$ BG, molecular weight $>1000 \mathrm{~kg} \mathrm{~mol}^{-1}$ and $0.25 \mathrm{~g} \mathrm{~mL}^{-1}$ density was provided by Tite \& Lyle PLC (London, UK). B-Can Oat Beta-Glucan with $70 \%$ BG, molecular weight between $100-200 \mathrm{~kg} \mathrm{~mol}^{-1}$ and density of $0.4-0.5 \mathrm{~g} \mathrm{mL^{-1 }}$ was provided by Garuda International, Inc. (Exeter, CA, USA) (data on molecular weight and density according to suppliers' information). The dietary fibre content was analysed in triplicate samples after enzymatic digestion with pepsin and pancreatin, centrifugation and dialysis of supernatants to obtain soluble (SDF) and insoluble dietary fibre (IDF) following the method modified in our laboratory; ${ }^{26}$ constituent neutral sugars and uronic acids were quantified spectrophotometrically after acid hydrolysis. The physicochemical properties (swelling, water-holding capacity and oil-holding capacity) of BG were analysed according to a study by Lecumberri et al. ${ }^{27}$ Viscosity was measured in the nutraceuticals on a Kinexus pro rheometer (Malvern Instruments Ltd; Malvern, Worcestershire, UK) equipped with a cone-plate of $60 \mathrm{~mm}$ diameter, $1^{\circ}$ angle, and a gap of $0.030 \mathrm{~mm}$ for $70 \%$ BG samples, or with a plate-plate of $40 \mathrm{~mm}$ diameter and a gap of $1.5 \mathrm{~mm}$ for $35 \% \mathrm{BG}$ samples. The samples were prepared by diluting $3 \mathrm{~g}$ and $5 \mathrm{~g}$ of $35 \% \mathrm{BG}$ and $70 \% \mathrm{BG}$, respectively, in $100 \mathrm{~mL}$ of distilled water. The temperature was controlled to be within $0.1^{\circ} \mathrm{C}$ by Peltier elements in the lower plate. A cover was used to maintain the samples at $37{ }^{\circ} \mathrm{C}$ and prevent evaporation. The apparent viscosity was measured at $37^{\circ} \mathrm{C}$ and a shear rate of $30 \mathrm{~s}^{-1}$ for $5 \mathrm{~min}$. During this time, the apparent viscosity remained constant, and an average value was obtained in accordance with previous studies carried out in BG solutions. ${ }^{28}$

Decaffeinated green coffee bean extract (GCBE). The source of hydroxycinnamates was a caffeine-free extract from green coffee provided by PharmaFoods S.L. (Barcelona, Spain). According to the technical specifications provided by the supplier, it contained approximately $45 \%(\mathrm{w} / \mathrm{w})$ PC, expressed as chlorogenic acids. The phenolic composition of the extract was analysed in our laboratory. Briefly, total soluble PC in the GCBE were extracted with $2 \mathrm{~N}$ hydrochloric acid in 50\% aqueous methanol ( $1 \mathrm{~h}$, room temperature) and centrifuged and the pellets were extracted with acetone/water $(70: 30, \mathrm{v} / \mathrm{v}$, $1 \mathrm{~h}$, room temperature). ${ }^{29}$ Supernatants from the two extractions were combined, concentrated and analysed by HPLC-DAD using an Agilent 1200 HPLC (Agilent Technologies, CA, USA) after injection in a Superspher RP18 column $(4.6 \mathrm{~mm}$ $\times 250 \mathrm{~mm}$ i.d., $4 \mu \mathrm{m}$; Agilent Technologies) as described by Gómez-Juaristi et $a .^{30}$

Nutraceuticals. The daily doses of BG products used were established based on the EFSA approved claims $\left(3 \mathrm{~g} \mathrm{~d}^{-1}\right.$ for lowering cholesterol and $4 \mathrm{~g}$ BG per $30 \mathrm{~g}$ of digestible carbohydrates to reduce postprandial glycaemia). Regarding the green coffee extract, the dose used was determined according to the previous results of human studies carried out by our research group using a blend of roasted/green coffee. 
Nutraceutical formulations were designed such that each study group consumed one of the four mixtures daily: low $\left(3 \mathrm{~g} \mathrm{~d}^{-1}\right)$ or high $\left(5 \mathrm{~g} \mathrm{~d}^{-1}\right)$ doses of BG, with either $35 \%$ or $70 \%$ concentration, and a fixed amount of GCBE providing $600 \mathrm{mg} \mathrm{d}^{-1} \mathrm{PC}$. The richness of the GCBE calculated in our laboratory $(14.8 \%$, see the Results section) and not that indicated by the manufacturer $(45 \%)$ was taken into account for the nutraceutical formulation to ensure the daily intake of $600 \mathrm{mg}$ PC. The formulations of the mixtures of BG and GCBE were prepared by QuimiFarma Laboratorios S.A. (Toledo, Spain) with different aroma compounds (chocolate, cappuccino, lemon and berries) for each formulation. Nutraceuticals were sealed in individual sachets that contained half of the daily dose, labelled A to D for blinding.

\subsection{Study design}

The present study followed a non-experimental pre-test/posttest design, in which the control is each individual at baseline. Bearing this in mind, a free-living randomised, dose-response, parallel, blind intervention was carried out. It was six weeks long and took place in spring. It was performed in 60 overweight/obese subjects, who were randomly allocated to one of the four groups $(n=15)$, designated according to the amount and type of BG: $3 \mathrm{~g}-35 \%, 5 \mathrm{~g}-35 \%, 3 \mathrm{~g}-70 \%$ and $5 \mathrm{~g}-70 \%$. The randomization of the study was carried out by one of the members of the research team, who generated random numbers in Excel, and it was controlled by sex. Initially we aimed at enrolling the same number of men and women in each group; however, more men agreed to participate. To reach the intake of BG and PC in GCBE established for the study, volunteers consumed the nutraceutical twice a day; the first dose was taken $30 \mathrm{~min}$ before breakfast and the second $30 \mathrm{~min}$ before lunch, always dissolved in $200 \mathrm{~mL}$ of water. Compliance was controlled by calling the volunteers every week and by counting the number of sachets provided to the volunteers before and after the intervention.

The study was conducted at the Human Nutrition Unit (HNU) of the Institute of Food Science, Technology and Nutrition (ICTAN) according to the guidelines laid down in the Declaration of Helsinki. All procedures were approved by the Ethics Committee of Hospital Universitario Puerta de Hierro Majadahonda in Madrid (Spain) and the Bioethics Committee of Consejo Superior de Investigaciones Científicas. The study was registered in ClinicalTrials (NCT04321590).

\subsection{Study subjects}

Volunteer recruitment was carried out by the research team through placing advertisements in the campus of the Universidad Complutense de Madrid, in health centres, research institutes, websites and nutrition forums. The inclusion criteria were male and female volunteers with BMI 25-35 kg m $\mathrm{m}^{-2}$, non-vegetarian, non-smoker, non-pregnant women, 18-60 years old, not suffering from any other chronic pathology or gastrointestinal disorder. Exclusion criteria included consuming dietary supplements, laxatives, or antibiotics six months before the start of the study. Sixty-two subjects initially accepted to participate in the study and gave informed written consent; however, for personal or health reasons, 2 subjects withdrew from the study and 60 completed it. Baseline characteristics of the volunteers are shown in Table 1. Although we aimed at sex parity during recruitment, finally more men than women participated in the study. Their level of education was medium or high. They were nonsmokers (in accordance with inclusion criteria), sedentary (see section 2.8), and many consumed alcohol, particularly at weekends.

\subsection{Dietary assessment}

Subjects were asked to maintain their dietary habits during the study. Participants completed a 72-hour food intake record before the baseline (handed in on week 1) and at the end (week 6) of the study. The dietary composition was assessed using the software DIAL (Departamento de Nutrición y Bromatología I. Facultad de Farmacia. Universidad Complutense de Madrid) which is based on the Spanish Food Composition Data Table reported by Mataix-Verdú et al. ${ }^{31}$

\subsection{Blood samples and biochemical analyses}

Blood samples were obtained by a nurse after 8-10 h overnight fasting at week 0 (baseline) and after consuming the nutraceutical for 6 weeks. Serum (without the anticoagulant) and plasma (EDTA-coated tubes) were separated by centrifugation $(15 \mathrm{~min}$ at $1750 \mathrm{~g})$ and frozen at $-20^{\circ} \mathrm{C}$ until analysis.

The lipid profile was determined in the serum samples using a Roche Cobas Integra 400 plus analyser (Roche Diagnostics, Madrid, Spain), following reference methods or methods recommended by Sociedad Española de Bioquimíca Clínica y Patología Molecular (SEQC). LDL-C was calculated as the difference between total and HDL-C. Glucose and the enzymes aspartate aminotransferase (ASAT) and alanine ami-

Table 1 Baseline characteristic of the volunteers who participated in the study

\begin{tabular}{|c|c|c|c|c|c|}
\hline & $3 \mathrm{~g}-35 \%(n=15)$ & $5 \mathrm{~g}-35 \%(n=15)$ & $3 \mathrm{~g}-70 \%(n=15)$ & $5 \mathrm{~g}-70 \%(n=15)$ & $p$ \\
\hline Age (y) & $39.4 \pm 2.7$ & $40.7 \pm 3.1$ & $35.7 \pm 3.5$ & $37.5 \pm 3.2$ & N.S. \\
\hline Weight (kg) & $80.3 \pm 2.7$ & $90.1 \pm 4.4$ & $88.1 \pm 5.0$ & $81.1 \pm 3.4$ & N.S. \\
\hline Height $(\mathrm{cm})$ & $166.6 \pm 2.2$ & $169.8 \pm 2.8$ & $168.4 \pm 2.8$ & $165.2 \pm 2.7$ & N.S. \\
\hline BMI $\left(\mathrm{kg} \mathrm{m}^{-2}\right)$ & $28.9 \pm 0.6$ & $31.2 \pm 1.3$ & $30.8 \pm 1.2$ & $29.8 \pm 0.9$ & N.S. \\
\hline DBP (mmHg) & $79.1 \pm 1.9$ & $81.1 \pm 1.7$ & $84.8 \pm 2.8$ & $79.1 \pm 2.7$ & N.S. \\
\hline $\mathrm{SBP}$ (mmHg) & $126.9 \pm 3.3$ & $127.2 \pm 2.1$ & $132.3 \pm 3.5$ & $125.2 \pm 4.0$ & N.S. \\
\hline Men/women & $11 / 4$ & $11 / 4$ & $11 / 4$ & $10 / 5$ & \\
\hline
\end{tabular}


notransferase (ALAT) were analysed according to standard spectrophotometric techniques.

\subsection{Blood pressure parameters}

Blood pressure was measured using an automatic arm sphygmomanometer (Pic Indolor Diagnostic, BS 150, Artsana, Italy). At the baseline and week 6, volunteers were asked to rest on a chair for 5 minutes before the cuff was placed on their left arm. After the participant rested for 5 minutes, a second reading was taken on their right arm. Readings were compared, and if not agreeing within $10-15 \mathrm{mmHg}$ a third reading was taken.

\subsection{Anthropometric measurements}

At the baseline and at the end of the study, volunteers' body weight, as well as total body water, body fat, visceral fat and segmental fat (arms, legs and trunk) were estimated using a Tanita BC-601 body composition monitor (Tanita Europe BV, Amsterdam, The Netherlands). To measure their height and body circumferences, measurement tape (Fisaude ADE, Madrid, Spain) and a Holtain precision mechanical stadiometer (Holtain Limited, Crymych, Dyfed, UK) were used.

\subsection{Physical activity, satiety and bowel habits}

Participants were asked to maintain their usual physical activity during the study. At the baseline and at the end of the intervention, on three representative days, volunteers filled out a questionnaire that has been previously used in our group ${ }^{13}$ to evaluate their physical activity, including that involving their occupation. Another questionnaire was used to evaluate the changes in satiety and bowel habits related to the consumption of the test products. Volunteers were asked to describe their perceptions of satiety, flatulence, bloating, intestinal movements, abdominal pain, soft stools, effort for a bowel movement, bowel movement, intestinal gas, hunger, heaviness and snaking between meals. Their answers were graded on a 1-5 scale or otherwise the following words were used: never, rarely, sometimes, frequently and always. Afterwards, for statistical treatment of the answers, the five appreciations were converted into a 1-5-point scale where $1=$ never, 2 = rarely, $3=$ sometimes, $4=$ frequently and $5=$ always. Regarding stool consistency, graduation went from $1=$ very soft up to $5=$ very hard.

\subsection{Statistical analysis}

The number of volunteers and the length of this study were established according to a similar parallel study, carried out in 12-16 volunteers per group, where significant effects of consuming a fibre supplement on body weight, BMI, body fat percentage, etc. were observed after 6 weeks. ${ }^{32}$ We established a sample size of at least 14 subjects per group taking body weight as the main variable, considering a standard deviation of 12 , aiming to detect an effect size of 0.537 , assuming a statistical power of $90 \%$ and a level of statistical significance of $5 \%$. Statistical analysis was performed using SPSS 23.0 for Windows (SPSS Inc., Chicago, IL, USA).
Data are expressed as means \pm standard errors unless specified otherwise. For all variables studied, the normality of distribution and the homogeneity of variance were evaluated using the Kolmogorov-Smirnov and Levene tests, respectively. To comparatively assess the response to the intervention, values obtained at the baseline and the end of the interventions were analysed using the General Linear Model with repeated measures, and the nutraceutical product was considered as an inter-individual factor. Afterwards, with the variables that showed statistical differences as far as the intervention $\times$ product, the rate of change was calculated as [(end value baseline value)/baseline value] and the Bonferroni correction paired $t$-test was carried out.

Data on satiety and bowel habits were expressed as means \pm standard deviation, and the results were analysed using the Kruskal Wallis test. As aforementioned, volunteers' answers were graded on a 1-5 scale or otherwise they answered: never, rarely, sometimes, frequently or always, and afterwards, their answers were converted as follows: $1=$ never, $2=$ rarely, $3=$ sometimes, $4=$ frequently and $5=$ always. Stool consistency was graded from $1-5$, so that $1=$ very soft up to $5=$ very hard.

\section{Results}

Overall, it is important to note that all volunteers completed the study, assisting to visits to the HNU and providing the different questionnaires. Therefore, there were no missing values in any of the sections 3.2,3.3, and 3.4.

\subsection{Characterisation of the beta-glucan products and the green coffee bean extract (GCBE)}

The physicochemical properties and composition of 35\% BG and $70 \%$ BG products were as follows: insoluble dietary fibre (\%): $6.1 \pm 0.6$ and $14.1 \pm 1.6$, respectively; soluble dietary fibre (\%): $24.2 \pm 2.4$ and $62.8 \pm 1.0$, respectively; moisture (\%) $5.0 \pm$ 0.1 and $4.0 \pm 0.3$, respectively; swelling capacity ( $\mathrm{mL}$ per $\mathrm{g} \mathrm{dm}$ sample): $6.1 \pm 0.5$ and $7.2 \pm 0.4$, respectively; water-holding capacity ( $g$ water per $g$ dm sample): $6.7 \pm 1.7$ and $8.03 \pm 1.07$, respectively; oil-holding capacity ( $\mathrm{g}$ oil per $\mathrm{g} \mathrm{dm}$ sample): $1.8 \pm$ 0.3 and $2.1 \pm 0.2$, respectively. As expected, the amount of DF was higher in $70 \%$ BG than in $35 \%$ BG, although both products contained remarkable amounts of IDF (about $20 \%$ of the total dietary fibre). According to the product data sheets provided by the manufacturer, $70 \%$ BG contained $\geq 70 \%$ BG determined by high performance liquid chromatography; however, according to the analysis carried out following the method described by Saura-Calixto et al., ${ }^{26}$ the content of SDF was lower, $62.8 \%$, together with $14 \%$ IDF. Likewise, according to the analysis carried out in our laboratory for $35 \%$ BG, this product presented a lower content of SDF, $24.2 \%$, along with $6 \% \mathrm{IDF}$. The higher SDF content in 70\% BG resulted in higher swelling capacity as well as higher water and oil retention capacities than those in $35 \%$ BG. In contrast, the viscosity was higher in the nutraceuticals prepared with $35 \%$ BG (1.60 and $5.50 \mathrm{~Pa} \mathrm{~s}^{-1}$ in the product containing 3 and $5 \mathrm{~g}$ of $\left.35 \% \mathrm{BG}\right)$ 
than that with $70 \%$ BG $\left(0.013\right.$ and $0.051 \mathrm{~Pa} \mathrm{~s}^{-1}$ in the nutraceuticals prepared with 3 and $5 \mathrm{~g}$ of $70 \% \mathrm{BG}$, respectively).

Quantification of PC in GCBE by HPLC-DAD showed that the content of phenolic compounds was much lower than that provided by the manufacturer, with only $14.8 \%$ (w/w) hydroxycinnamic acids. Monoacylquinic acid derivatives represented $76.1 \%$ of the total PC in GCBE, constituted mainly of caffeoylquinic acids (67.9\%), feruloylquinic acids (7.9\%), and coumaroylquinic acids $(0.3 \%)$. The next most abundant group was diacylquinic acids $(22.4 \%$ of total polyphenols), formed by dicaffeoylquinic acids (16.7\%), caffeoylferuloylquinic acids (4.3\%), and coumaroylcaffeoylquinic acids (1.4\%). Minor amounts of caffeoyl- $\mathrm{N}$-tryptophan, caffeoyl-glycosides and caffeic acid $(1 \%, 0.3 \%$ and $0.1 \%$ of total PC, respectively) were also detected.

\subsection{Dietary assessment}

The macronutrient daily intake is shown in Table 2. There were no significant differences in the energy intake with any of the nutraceutical treatments between the baseline and the end of the intervention. Volunteers' caloric consumption remained between 2238-2543 $\mathrm{kcal} \mathrm{day}^{-1}$, thus they maintained their usual energy intake. There were no differences in the macronutrient intake except for carbohydrates that significantly decreased after the intervention without showing differences between the nutraceutical products studied. The reductions in nutrient intake observed, without variations in the energy intake, suggest that volunteers may have changed their food choices, which may be related to the intake of the nutraceutical treatments and, to a certain extent, it may also be associated with the differences in how carefully the volunteers filled out the dietary records at the beginning and the end of the interventions. This is explained in more detail in the paragraph about the limitations of the study.

\subsection{Effects on cardiovascular health, lipid and glucose metabolism, and blood pressure}

At the beginning of the study, volunteers' blood T-C levels were not far from the limit of hypercholesterolemia $\left(200 \mathrm{mg} \mathrm{dL}^{-1}\right)$, whereas LDL-C, very low density lipoprotein cholesterol (VLDL-C) and TG levels were within the healthy range (Table 3$)$. The concentration of T-C $(p=0.001)$, LDL-C $(p=$ $0.001)$, VLDL-C ( $p=0.044)$, and TG $(p=0.023)$ significantly decreased at the end of the study compared to the baseline. In turn, there were no differences between the groups that consumed the nutraceuticals. Both ASAT and ALAT enzymes decreased at the end of the intervention $(p=0.023$ and $p=$ 0.010, respectively) (Table 3), again without differences between the products studied. In contrast, the HDL-C levels did not change after the intervention.

Although volunteers showed overweight or obesity, they were not pre-diabetic according to the baseline levels of parameters related to glucose metabolism. The fasting glucose concentration was under $100 \mathrm{mg} \mathrm{mL}^{-1}$ and remained within the healthy range, although the values increased $(p=0.044)$ at the

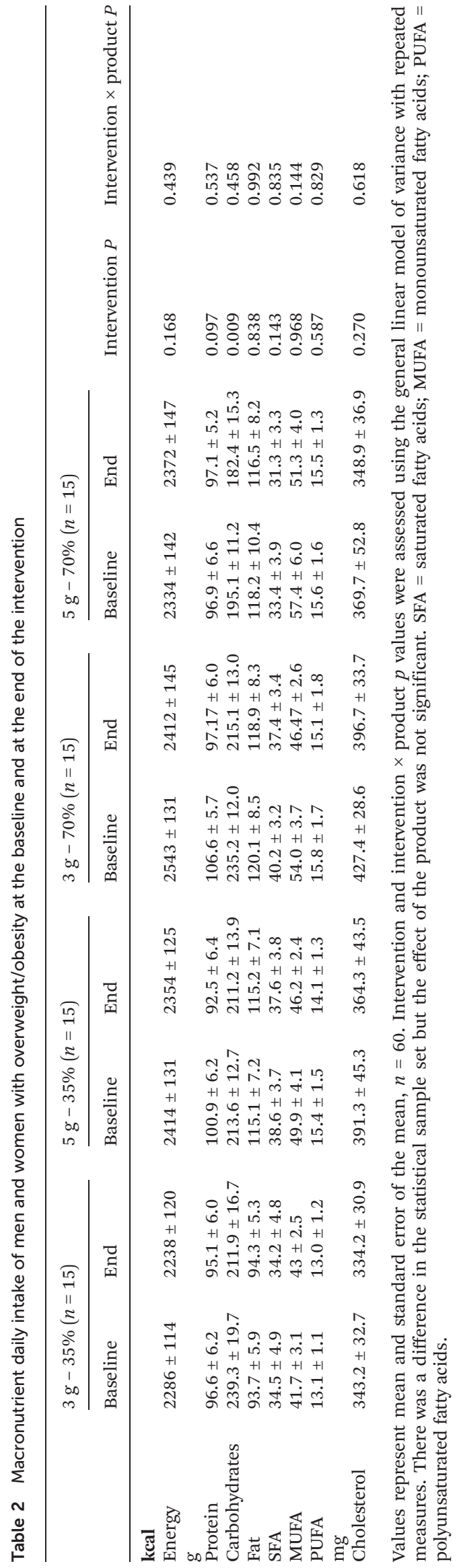

This journal is (c) The Royal Society of Chemistry 2022 


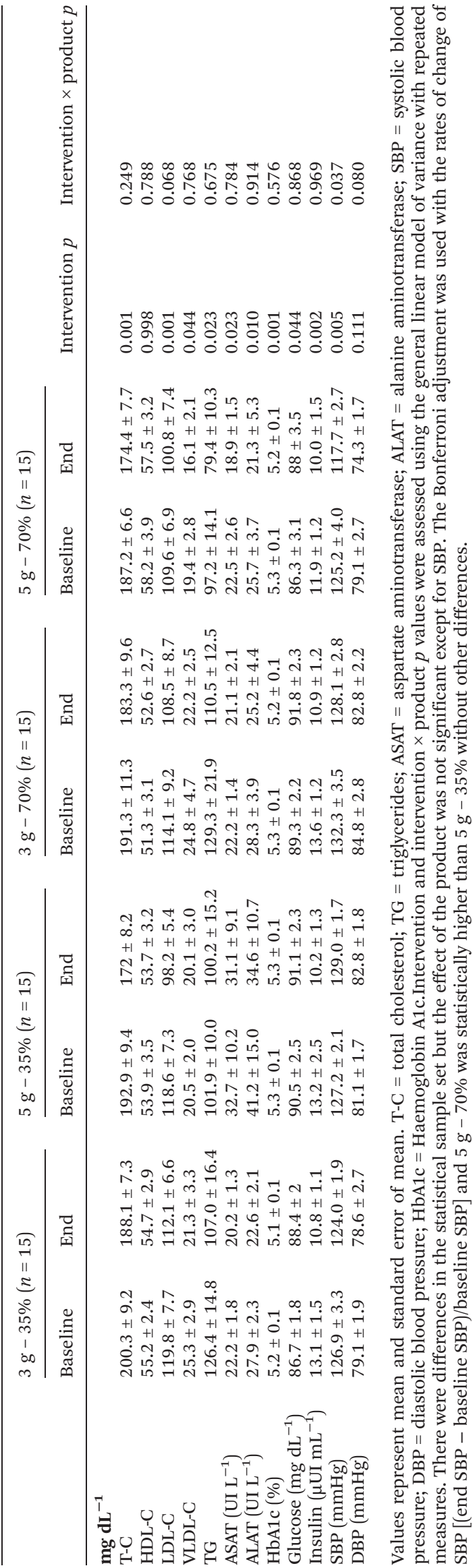

end the study. In contrast, after the intervention, the glycosylated haemoglobin (HbA1c, $p=0.001)$ and insulin $(p=0.002)$ concentration significantly decreased, without differences among the nutraceuticals (Table 3).

All the volunteers who participated in the study were normotensive [systolic blood pressure (SBP) $<140 \mathrm{mmHg}$, diastolic blood pressure (DBP) $<90 \mathrm{mmHg}$. Interestingly, after the intervention SBP values were significantly lower, and the intervention $\times$ product was significant $(p=0.037)$; in contrast, the DBP remained unchanged along the study. When the rate of change of SBP was calculated, $5 \mathrm{~g}-70 \%$ BG showed the highest reduction, being statistically different from $5 \mathrm{~g}-35 \%$ BG according to the Bonferroni test.

\subsection{Effects on anthropometric parameters, subjective satiety and bowel habits}

After the intervention, no significant changes in body weight or BMI (Table 4) were observed, neither differences between the products studied. In contrast, the body fat percentage $(p=$ $0.009)$ significantly decreased after the intervention, as well as fat in different compartments: visceral fat percentage ( $p=$ $0.001)$, trunk fat percentage $(0.001)$, and right $(p=0.014)$ and left arm fat percentage $(p=0.002)$. Likewise, waist $(p<0.001)$ and hip $(p<0.001)$ circumferences significantly decreased after the consumption of the four nutraceuticals (Table 4). In turn, there were no differences between the groups that consumed the nutraceuticals, except for TBF\% (intervention $\times$ product $p=0.022$, Table 4 ). Therefore, the Bonferroni adjustment was used only with the rate of change of $\mathrm{TBF} \%$, and we observed that with the rate of change of TBF\%, $5 \mathrm{~g}-70 \%$ was statistically lower than $3 \mathrm{~g}-70 \%$, without differences between the other groups. Attending to the FAO/WHO/UNU Expert Consultation Report on Energy and Protein Requirements (1985), volunteers' physical activity was moderate and it did not show modifications along the study (data not shown).

Subjective perceptions on the effects of consuming the nutraceuticals on satiety (Fig. 1) and bowel habits (Table 5), described by volunteers at the end of the intervention, showed no differences among the products. Only bloating $(p=0.045)$ was statistically lower after the $5 \mathrm{~g}-70 \%$ BG treatment, and intestinal gas was close to higher with $5 \mathrm{~g}-35 \%$ BG $(p=0.065)$.

\section{Discussion}

In the present study, we comparatively evaluated the effects on obesity and its associated comorbidities of nutraceutical formulations containing oat BG products with different concentrations (35\% versus $70 \%)$ at two doses $\left(3 \mathrm{~g} \mathrm{~d}^{-1}\right.$ or $\left.5 \mathrm{~g} \mathrm{~d}^{-1}\right)$, together with a fixed amount of GCBE providing $600 \mathrm{mg} \mathrm{d}^{-1}$ PC, as a dietary supplement in free living men and women with overweight or type 1 obesity.

Of the BG products used in the present study, 70\% BG was twice as concentrated as $35 \%$ BG, although its molecular 


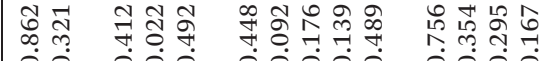

weight was about ten times lower $\left(100-200 \mathrm{~kg} \mathrm{~mol}^{-1}\right.$ for $70 \%$ BG vs. $>1000 \mathrm{~kg} \mathrm{~mol}^{-1}$ for $35 \%$ BG, according to the information provided by the suppliers). We are not aware of the procedure used by the manufacturers to obtain the BG products nor in which stages might changes in the molecular structure of the BG have occurred, from the extraction of the polymer from oat tissues to the concentration of the fibre to obtain the final purified product. This might be especially relevant in $70 \%$ BG, considering its higher concentration and lower molecular weight.

In general, soluble fibre form viscous solutions due to their high hydration capacity, with high water-holding and swelling capacities. According to our results, 70\% BG showed slightly higher hydration capacity than $35 \% \mathrm{BG}$, as its swelling capacity $\left(7.2 \pm 0.4\right.$ vs. $\left.6.1 \pm 0.5 \mathrm{~mL} \mathrm{~g}^{-1}\right)$ and water-holding capacity (8.03 \pm 1.07 vs. $6.74 \pm 1.66 \mathrm{~g}$ water per $\mathrm{g}$ BG) were greater. Compared to other soluble fibre, the swelling capacity of the two BG was marginally lower than that of apple $\left(7.42 \pm 1.15 \mathrm{~mL} \mathrm{~g}^{-1}\right)$ and citrus pectins $\left(10.45 \pm 1.21 \mathrm{~mL} \mathrm{~g}^{-1}\right)$. Similarly, their water holding capacity was noticeably lower than that of apple and citrus pectins ( $16.51 \pm 3.77$ and $28.07 \pm 5.34 \mathrm{~g}$ water per $\mathrm{g}$ SDF, respectively), as described by Lecumberri et al. in $2007 .^{27}$ Concerning viscosity, although this was analysed in the formulated nutraceuticals instead of the pure BG, higher viscosity was observed in the products containing $35 \%$ BG, with values 100 times higher than those of the products containing $70 \%$ BG. In addition, the dose of BG also affected the viscosity of the nutraceutical, since the $5 \mathrm{~g}$ dose was about 3.4 to 3.9 times more viscous than the $3 \mathrm{~g}$ dose.

The functionality of BG is mainly dependent on their molecular weight, type of linkage (presence of mixed $\beta-(1 \rightarrow 4)$ and $\beta-(1 \rightarrow 3)$ links), and chain length. It is still challenging to obtain pure BG extracts with high concentration and molecular weight, as generally these may produce greater positive health effects. However, high molecular weight structures may have lower solubility and even inhibit some physiological functions in vivo. ${ }^{11,33}$ On the other hand, low molecular weight $\mathrm{BG}$ have advantages such as not causing undesirable sensory properties, slowing down filtration of solutions, or precipitation, thus extending their applications in the food and pharmaceutical industries. ${ }^{34}$ Nevertheless, some studies have shown that modifying BG molecular weight reduced their antihypercholesterolemic properties, ${ }^{35}$ reducing their efficacy in lowering LDL-C concentrations, ${ }^{36}$ glycaemic and insulinemic responses, ${ }^{37}$ and body weight. ${ }^{38}$ In contrast, consuming high or low molecular weight BG did not lead to differences in blood lipids, insulin or glucose levels in another human study, ${ }^{39}$ thus the interest in studying the two BG products used in the present intervention trial was shown.

In addition to the physicochemical properties of $\mathrm{BG}$, dosage also plays an important role in the biological effects of SDF. The EFSA and the Food and Drug Administration (FDA) approved that $3 \mathrm{~g}$ BG per day could have a positive effect on reducing cholesterol levels. ${ }^{6}$ However, processing that degrades BG may result in less effective products, and thus, the recommended intake of $>3 \mathrm{~g} \mathrm{day}^{-1}$ of BG does not ensure a sig- 


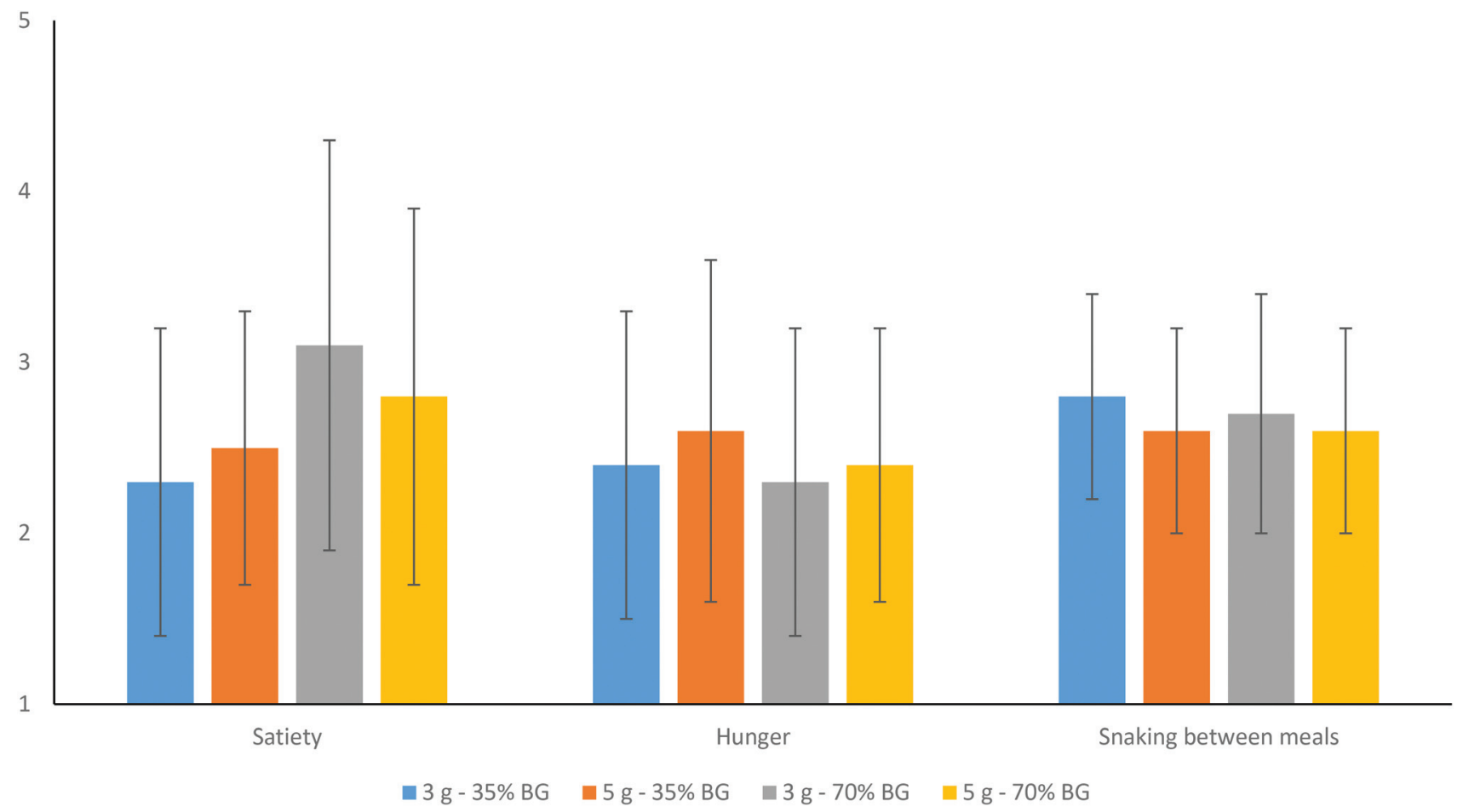

Fig. 1 Effects of consuming the nutraceutical with 35\% BG and 70\% BG at $3 \mathrm{~g} \mathrm{day}^{-1}$ and $5 \mathrm{~g} \mathrm{day}^{-1}$ for 6 weeks on the subjective perception of satiety, hunger and snacking in men and women with overweight/obesity. Marks are between 1 (null) to 5 (maximum) points scale.

Table 5 Effects of consuming the nutraceutical with 35\% BG and 70\% BG at $3 \mathrm{~g}$ day $^{-1}$ and $5 \mathrm{~g} \mathrm{day}^{-1}$ for 6 weeks on intestinal habits in men and women with overweight/obesity

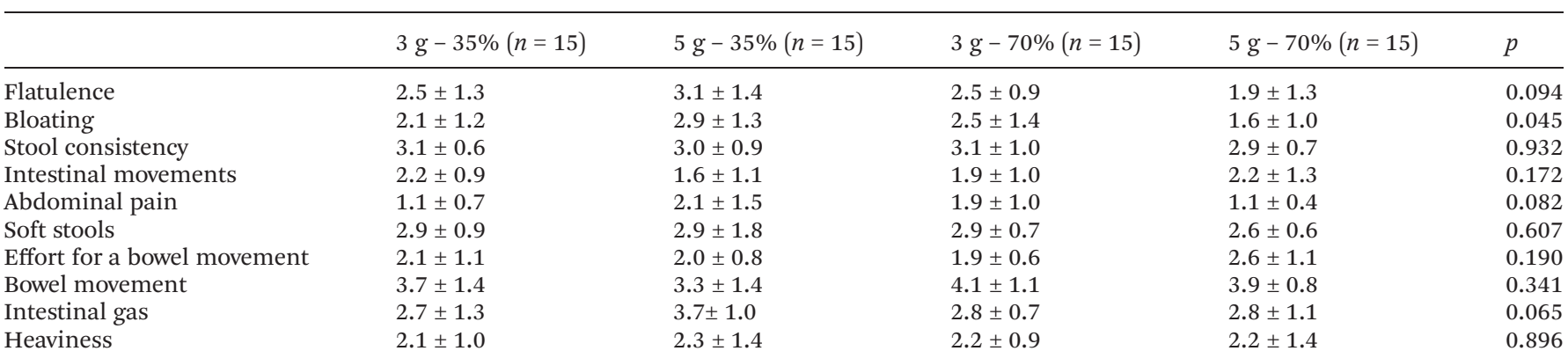

Values represent mean and standard deviation. Answers were graded on a 1-5 scale or otherwise the following words used: never, rarely, sometimes, frequently and always, and afterwards were converted into a $1-5$-point scale where $1=$ never, $2=$ rarely, $3=$ sometimes, $4=$ frequently and $5=$ always. Regarding stool consistency graduation went from $1=$ very soft up to $5=$ very hard. $P$ values were assessed using the KruskalWallis test.

nificant decrease in blood cholesterol. ${ }^{3,40}$ A recent metaregression analysis did not find a dose-response relationship between barley BG intake and their cholesterol lowering effect. ${ }^{41}$ In turn, in the meta-analysis just published by Zurbau et $a l .,{ }^{37}$ the magnitude of glucose reduction by oat BG depended on the dose, along with the molecular weight.

In the present study, when the pre-test and post-test results were compared, a general improvement in cardiometabolic parameters was observed in the free-living participants who consumed their habitual diet, as T-C $(p=0.001)$, LDL-C $(p=0.001)$ and, to a lower extent, VLDL-C $(p=0.044)$ and TG $(p=0.023)$, significantly decreased, whereas there were no differences in HDL-C concentrations (Table 3 ). The positive effects of the nutraceuticals studied on T-C, LDL-C and TG are in agreement with previous studies on $\beta$-glucans ${ }^{3,42}$ as well as the absence of the effects on HDL-C with that reported in the meta-analysis by Ho et $a l^{41}$ The results here obtained are novel and relevant, as few studies have evaluated the effect of oat $\beta$-glucan products, including their physicochemical properties, on the size and concentration of lipoprotein particles. ${ }^{43}$ Clearly, the dose of BG plays an important role in the reduction of LDL-C levels, since always the $5 \mathrm{~g}$ dose had higher 
effects than the lower one. Out of the four nutraceuticals, $5 \mathrm{~g}$ $35 \%$ BG had slightly higher, although statistically not significant, LDL-C decreasing effect, which may be related to its higher molecular weight and viscosity, as mentioned above. Therefore, these results support that the dose and physicochemical properties of BG may influence their LDL-C lowering properties in agreement with previous studies. ${ }^{36}$ In this context, it was interesting to see that the nutraceuticals containing low-molecular weight $70 \%$ BG showed similar LDL-C reducing effects to that containing 35\% BG, as $70 \%$ BG shows physicochemical properties that may allow many applications in the food industry. ${ }^{34}$ It is well known that LDL-C is a strong predictor of cardiovascular disease (CVD) and it can provide an independent measure of atherogenicity. ${ }^{44}$ The results here observed with the four treatments have important implications for the public health burden of coronary heart disease (CHD) as each $1 \%$ reduction in LDL-C reduces the coronary artery disease (CAD) risk by $1-2 \%$, and $1 \%$ decrease in T-C represents a $2-3 \%$ lowering in $\mathrm{CAD}$ risk. ${ }^{45}$ Therefore, the magnitude of the LDL-C reduction observed in the present study with the nutraceuticals would translate into a range of $17.2-4.8 \%$ decrease in CHD risk.

Soluble dietary fibre from oats also plays an important role in the prevention of type 2 diabetes, as generally SDF improves glucose and insulin responses in normoglycaemic and diabetic subjects. ${ }^{38}$ However, there is a controversy regarding the effects of oat BG on glucose metabolism parameters in longterm studies, and only a few trials have considered the physicochemical properties of BG. ${ }^{41}$ Several reviews based on longterm interventions have concluded that oats and oat bran do not affect fasting glycaemia, insulin concentration, insulin resistance or sensitivity, ${ }^{43}$ nor HbA1c and fasting glucose in subjects with type 2 diabetes. ${ }^{46}$ Contrarily, according to a systemic review and meta-analysis of studies carried out in subjects with type 2 diabetes, the consumption of $2.5-3.5 \mathrm{~g}^{-1 a y}{ }^{-1}$ of BG significantly lowered HbA1c by $0.21 \%$ and fasting glucose by $0.52 \mathrm{mmol} \mathrm{L}^{-1}$ without affecting plasma insulin concentrations. ${ }^{47}$ In a recent review and meta-analysis, it was concluded that adding BG to carbohydrate-containing meals reduces glycaemic and insulinemic acute responses regardless of the health status, and the magnitude of the reduction depends on the $\mathrm{BG}$ dose and molecular weight. ${ }^{37}$ Bearing in mind that this study followed a pre-test/post-test design, this work supports that the regular consumption of nutraceuticals containing $35 \%$ BG and $70 \%$ BG consumed at 3 and $5 \mathrm{~g} \mathrm{day}^{-1}$, combined with the green coffee extract, produces positive effects on glucose metabolism parameters, reducing HbA1c and insulin levels, although, contrary to the conclusions reported by Zurbau et al., ${ }^{37}$ there were no differences due to the molecular weight or dose of the BG studied. In contrast, the glucose concentration increased at the end of the intervention, although the $p$ value obtained was near the level of statistical significance $(p=0.044)$ (Table 3$)$, and the glycaemic concentration remained within the healthy range. Regarding the possible mechanisms involved in these effects, it is well known that viscosity may play a key role in modulating gastric emptying, inhibiting carbohydrate digestion and decreasing glucose absorption. However, the lack of differences observed between $35 \%$ BG and $70 \%$ BG despite the higher viscosity of the former suggests that other mechanisms might have been responsible for the observed changes, such as the modulation of the gastrointestinal microbiome. ${ }^{48}$

Epidemiological studies suggest an inverse association between dietary fibre intake and blood pressure. However, according to clinical trials, this relationship is not clear as some studies have pointed to a small anti-hypertensive effect of fibre supplementation, whereas others show no effect. ${ }^{38}$ In the review by Thies et al., ${ }^{43}$ few studies described a significant effect of increased oat consumption on blood pressure, but this result was partly attributed to the studies not being adequately powered and the differences in the methodology used to measure the blood pressure. Nevertheless, three studies supported the decreases in systolic blood pressure between $4 \%{ }^{49,50}$ and $6 \% .{ }^{51}$ Moreover, in a randomized controlled study carried out in healthy middle-aged subjects, a reduction in systolic blood pressure was observed when 3 daily portions of whole grain wheat or a mixture of wheat and oats were consumed for 12 weeks in comparison to consuming a refined cereal. $^{52}$ Accordingly, in the present work a significant reduction in systolic blood pressure $(p=0.005)$ was observed after the consumption of the nutraceuticals for six weeks. Attending to the rates of change of SBP, among the treatments studied, $5 \mathrm{~g}-70 \%$ BG was more effective than $5 \mathrm{~g}-35 \%$ BG treatment. To our knowledge, this is the first study that looks into the physicochemical properties of BG in relation to its effects on blood pressure.

As far as the effects of consuming the nutraceuticals on anthropometric parameters are concerned, there were statistical differences for total body water $(p=0.001)$, TBF\% $(p=$ $0.009)$, and visceral fat $(p=0.001)$, as well as waist $(p<0.001)$, hip $(p<0.001)$, right arm $(p=0.001)$, and thigh circumferences $(p=0.001)$, and only TBF\% showed statistical differences among the products. Taking into account the rates of change of $\mathrm{TBF} \%$, a greater reduction in $\mathrm{TBF} \%$ was observed with $5 \mathrm{~g}-$ $70 \%$ BG that was significantly different from $3 \mathrm{~g}-70 \%$ BG according to the Bonferroni paired test, which may be related to the low molecular weight BG being more fermentable than $35 \%$ BG. In a recent study carried out in mice, ${ }^{53}$ changes in abdominal fat depots, serum cholesterol and leptin concentrations in the group of animals that consumed the low molecular weight BG were related to BG prebiotic effects, as almost $100 \%$ was fermented and caecal bacterial counts of Bifidobacterium and Bacteroides significantly increased, increasing the caecal contents of acetic and propionic acids. In contrast, in mice fed the high molecular weight BG, the positive changes in abdominal fat, serum cholesterol and leptin concentrations observed were related to the inhibition of nutrient absorption due to high viscosity in the digestive tract. In addition, the reduction in the rate of gastric emptying and nutrient absorption and the increase in the fermentation of soluble fibre producing short chain fatty acids (SCFAs) may lead to a decrease in fat deposits and an increase in fat oxi- 
dation, and thus reduction in body weight. ${ }^{54}$ In the present study, we did not analyse the potential changes in the intestinal microbiota nor in the production of SCFAs as a marker of the BG's fermentation, and thus it cannot be established which of these mechanisms might explain the observed reduction in total body and visceral fat, and body fat compartments as well as waist circumferences.

On the other hand, previous research ${ }^{55}$ supports that dietary fibre may reduce the energy intake because of increased satiety, leading to weight loss. Two human intervention studies assessed the effects of oat or barley products on appetite ratings (including satiety) after eating the test food on a single occasion, ${ }^{56,57}$ although only the former reported the effects on subsequent energy intake. ${ }^{53}$ It is noteworthy that these studies focused on single meals and not on the effects of the sustained consumption of fibre rich products, such as in this study that approached the influence on appetite as well as on subjective digestive function related to consuming twice a day the nutraceuticals containing BG for six weeks. This is an important issue, as effects on satiety may continue up to several hours and reduce the energy intake at the next meal or, if sustained across the day, it may lead to a greater reduction in food intake and thus body weight. According to volunteers' answers on the perception of hunger, heaviness and snacking between meals, marks were between 2.1 and 2.7 on a 5 points scale (Fig. 1). This suggests that their overall perceptions were moderate, and thus no significant differences between the treatments were found at this level. In addition, the fact that the energy intake did not change along the study with any of the nutraceuticals (Table 2) supports the overall lack of effect on satiety. This also points to other effects different from increased satiety and reduction in energy intake to explain the positive changes observed in body fat percentages and body circumferences.

It is likely that with the intake of the nutraceuticals, particularly those that contained $35 \%$ BG, the viscosity of the chyme may have increased, slowing down the intestinal transit and thus affecting the appetite for high density food, ${ }^{9,10}$ which may be related to the lower intake of carbohydrates after the intervention. However, controversial effects of BG and, in general, dietary fibre on macronutrient intake have been described, as not all studies have shown such a decrease. ${ }^{55,58,59}$ It seems that other factors, such as subject's BMI, etc. would influence the food intake. Moreover, the duration of the intervention seems to play an important role. ${ }^{55}$ Nevertheless, the change in carbohydrate intake did not translate into differences in energy intake with any of the tested nutraceuticals.

The nutraceuticals were well accepted by the volunteers and no adverse effects were reported. Concerning bowel habits, there was only a difference in the sensation of bloating ( $p=$ 0.045 ) and marginally in the discomfort produced by intestinal gas $(p=0.065)$, with $5 \mathrm{~g}-35 \%$ BG showing higher values (Table 5). This outcome may be related to the 100-times higher viscosity of $35 \%$ BG compared to $70 \%$ BG, combined with the higher amount $\left(5 \mathrm{~g} \mathrm{day}^{-1}\right)$ consumed. It also points to a higher fermentation of $35 \%$ BG. However, these results contrast with previous studies in which BG have shown good tolerance, not causing adverse gastrointestinal symptoms with daily intakes of up to $10 \mathrm{~g},{ }^{60}$ although Rebello et $a{ }^{48}{ }^{48}$ also reported higher flatulence in subjects with overweight/obesity that consumed a product containing $8.8 \mathrm{~g}$ of dietary fibre (including $2.5 \mathrm{~g}$ of oat BG) daily for four weeks.

This study presents certain limitations. Firstly, there was no control group, so the effect of the intervention itself, rather than the nutraceutical treatments, cannot be separately identified. In addition, no nutraceutical containing only BG, without GCBE, was used to understand the effect of BG and to clarify the contribution of the PC in GCBE. Similarly the fixed amount of GCBE in the nutraceuticals tested (600 $\left.\mathrm{mg} \mathrm{d}^{-1}\right)$ could have contributed to the positive effects observed on lipid, glucose and anthropometric parameters, as well as on blood pressure, in agreement with the previous studies carried out in our group; ${ }^{13,61}$ however, the extent of this contribution cannot be determined. Regarding BG, the actual degree of polymerization of the two BG products used was not studied, nor the viscosity of the pure BG. Another limitation was that the dietary analysis was based on $72 \mathrm{~h}$ records that were filled out three days prior to the two visits to the Human Nutrition Unit; no food preference or food-frequency questionnaires were considered. In addition, due to time constraints, it was not possible to instruct the volunteers on how to fill in the $72 \mathrm{~h}$ records on the home standard measurements and servings, thus it is not possible to rule out under- or overestimation of food intake by volunteers. Satiety was analysed through subjective measurements, caecal or faecal samples were not collected, and thus the analysis of volunteers' microbiota was not carried out to study the potential involvement of the colonic microbiota in the observed outcomes.

\section{Conclusion}

The regular consumption of the four nutraceuticals based on the combination of oat BG and PC from GCBE may reduce the cardiovascular risk and help in preventing type 2 diabetes and obesity. Among the nutraceutical treatments, there were minor differences; however, $5 \mathrm{~g}-70 \%$ BG induced slightly greater slimming effects, as it was the product that lowered $\mathrm{TBF} \%$ the most and additionally, it produced the least bloating according to participants' subjective perception. The slimming effect is likely to be related to mechanisms other than satiety, according to subjective questionnaires and energy intake regulation.

\section{Author contributions}

Conceptualization: LB, RM and BS; data curation: BS and JG-C; funding acquisition: $\mathrm{LB}$ and $\mathrm{RM}$; investigation: $\mathrm{BS}, \mathrm{RM}$ and LB; methodology: RM and BS; project administration: LB, BS and RM; resources: RM, LB and BS; supervision: BS and RM; writing - original draft: BS and JG-C; writing - review \& editing: BS, RM and LB. 


\section{Conflicts of interest}

There are no conflicts to declare.

\section{Acknowledgements}

The authors want to thank the volunteers who participated in the study, Dr. María Dolores Alvarez for carrying out viscosity analysis, Maria Yaguas and Elena Tocino for their assistance in dietary record analysis and volunteer recruitment, and Isabel Fernández-Conde for dietary fibre analysis, including physicochemical properties. This research was funded by project AGL2015-69986-R from the Ministerio de Economía y Competitividad. JGC had a predoctoral grant funded by Comunidad de Madrid (PEJD-2018-PRE/SAL-9104). Written in memory of Henry H. Sarria.

\section{References}

1 Obesity and overweight, 2019, Retrieved from https://www. who.int/news-room/fact-sheets/detail/obesity-andoverweight.

2 G. A. Bray, K. K. Kim and J. P. H. Wilding, World Obesity Federation, Obesity: a chronic relapsing progressive disease process. A position statement of the World Obesity Federation, Obes. Rev., 2017, 18, 715-723.

3 Q. Wang and P. R. Ellis, Oat $\beta$-glucan: physico-chemical characteristics in relation to its blood-glucose and cholesterol-lowering properties, Br. J. Nutr., 2014, 112, S4-S13.

4 J. García-Cordero, B. Sarriá-Ruiz, S. González-Rámila, L. Bravo-Clemente and R. Mateos-Briz, Effectiveness of hydroxycinamates and beta-glucans as dietary tools against obesity and its associated dysfunctions, and their application as nutraceuticals, Nutr. Hosp., 2020, 37, 1061-1071.

5 J. L. Bresson, A. Flynn, M. Heinonen, K. Hulshof, H. Korhonen, P. Lagiou, M. Løvik, R. Marchelli, A. Martin, B. Moseley, H. Przyrembel, S. Salminen, S. Strain, S. Strobel, I. Tetens, H. van den Berg, H. van Loveren and H. Verhagen, Scientific Opinion on the substantiation of health claims related to beta glucans and maintenance of normal blood cholesterol concentrations (ID 754, 755, 757, $801,1465,2934)$ and maintenance or achievement of a normal body weight (ID 820, 823) pursuant to Article 13(1) of Regulation (EC) No 1924/2006, EFSA J., 2009, 7, 1254.

6 C. Agostoni, J. L. Bresson, S. J. Fairweather-Tait, A. Flynn, I. Golly, H. Korhonen, P. Lagiou, M. Løvik, R. Marchelli, A. Martin, B. Moseley, M. Neuhäuser-Berthold, H. Przyrembel, S. Salminen, S. Sanz, S. Strain, S. Strobel, I. Tetens, D. Tomé, H. van Loveren and H. Verhagen, Scientific Opinion on the substantiation of a health claim related to oat beta glucan and lowering blood cholesterol and reduced risk of (coronary) heart disease pursuant to Article 14 of Regulation (EC) No 1924/2006, EFSA J., 2011, 9, 2471.
7 R. Bozbulut and N. Sanlier, Promising effects of $\beta$-glucans on glyceamic control in diabetes, Trends Food Sci. Technol., 2018, 83, 159-166.

8 C. Agostoni, J. L. Bresson, S. J. Fairweather-Tait, A. Flynn, I. Golly, H. Korhonen, P. Lagiou, M. Løvik, R. Marchelli, A. Martin, B. Moseley, M. Neuhäuser-Berthold, P. Przyrembel, S. Salminen, Y. Sanz, S. Strain, S. Strobel, I. Tetens, D. Tomé, H. van Loveren and H. Verhagen, Scientific Opinion on the substantiation of health claims related to beta-glucans from oats and barley and maintenance of normal blood LDL-cholesterol concentrations (ID $1236,1299)$, increase in satiety leading to a reduction in energy intake (ID 851, 852), reduction of post-prandial glycaemic responses (ID 821, 824), and "digestive function" (ID 850) pursuant to Article 13(1) of Regulation (EC) No 1924/2006, EFSA J., 2011, 9, 2207.

9 D. El Khoury, C. Cuda, B. L. Luhovyy and G. H. Anderson, Beta glucan: health benefits in obesity and metabolic syndrome, J. Nutr. Metab., 2012, 851362.

10 S. Pentikäinen, L. Karhunen, L. Flander, K. Katina, A. Meynier, P. Aymard and K. Poutanen, Enrichment of biscuits and juice with oat $\beta$-glucan enhances postprandial satiety, Appetite, 2014, 75, 150-156.

11 Y. Wang, N. P. Ames, H. M. Tun, S. M. Tosh, P. J. Jones and E. Khafipour, High molecular weight barley $\beta$-glucan alters gut microbiota toward reduced cardiovascular disease risk, Front. Microbiol., 2016, 7, 129.

12 L. Bravo, Polyphenols: chemistry, dietary sources, metabolism and nutritional significance, Nutr. Rev., 1998, 56, 317-333.

13 S. Martínez-López, B. Sarriá, R. Mateos and L. BravoClemente, Moderate consumption of a soluble green/ roasted coffee rich in caffeoylquinic acids reduces cardiovascular risk markers: results from a randomized, crossover, controlled trial in healthy and hypercholesterolemic subjects, Eur. J. Nutr., 2018, 58, 865-878.

14 S. Yu, S. W. Yue, Z. Liu, T. Zhang, N. Xiang and H. Fu, Yerba mate (Ilex paraguariensis) improves microcirculation of volunteers with high blood viscosity: A randomized, double-blind, placebo-controlled trial, Exp. Gerontol., 2015, 62, 14-22.

15 M. A. Alam, N. Subhan, H. Hossain, M. Hossain, H. M. Reza, M. M. Rahman and M. O. Ullah, Hydroxycinnamic acid derivatives: a potential class of natural compounds for the management of lipid metabolism and obesity, Nutr. Metab., 2016, 13, 27.

16 G. A. Klein, A. Stefanuto, B. C. Boaventura, E. C. De Morais, L. D. S. Cavalcante, F. de Andrade, E. Wazlawik, P. F. Di Pietro, M. Maraschin and E. L. Da Silva, Mate tea (Ilex paraguariensis) improves glycemic and lipid profiles of type 2 diabetes and pre-diabetes individuals: a pilot study, J. Am. Coll. Nutr., 2011, 30, 320-332.

17 J. Blum, B. Lemaire and S. Lafay, Effect of a green decaffeinated coffee extract on glycaemia, Nutr. Foods Res., 2007, 6, 13-17.

18 B. J. Peng, Z. H. U. Qi, Y. L. Zhong, S. H. Xu and W. Zheng, Chlorogenic acid maintains glucose homeostasis through 
modulating the expression of SGLT-1, GLUT-2, and PLG in different intestinal segments of Sprague-Dawley rats fed a high-fat diet, Biomed. Environ. Sci., 2015, 28, 894-903.

19 J. Santana-Gálvez, L. Cisneros-Zevallos and D. A. JacoboVelázquez, Chlorogenic acid: Recent advances on its dual role as a food additive and a nutraceutical against metabolic syndrome, Molecules, 2017, 22, 358.

20 A. Gambero and M. Ribeiro, The positive effects of yerba maté (Ilex paraguariensis) in obesity, Nutrients, 2015, 7, 730-750.

21 M. Jaquet, I. Rochat, J. Moulin, C. Cavin and R. Bibiloni, Impact of coffee consumption on the gut microbiota: a human volunteer study, Int. J. Food Microbiol., 2009, 130, 117-121.

22 H. Roshan, O. Nikpayam, M. Sedaghat and G. Sohrab, Effects of green extract supplementation on anthropometric indices, glycaemic control, blood pressure, lipid profile, insulin resistance and appetite in patients with the metabolic syndrome: a randomised clinical trial, Br. J. Nutr., 2018, 119, 250-258.

23 Z. Gorgi, H. K. Varkaneh, S. Talaei, A. Nazary-Vannani, C. C. T. Clark, Z. Fatahi, J. Rahmani, S. Salamat and Y. Zhang, The effect of green-coffee extract supplementation on obesity: a systematic review and dose-response meta-analysis of randomized controlled trials, Phytomedicine, 2019, 63, 153018.

24 M.-H. Pan, Y.-C. Tung, G. Yang, S. Li and C.-T. Ho, Molecular mechanisms of the anti-obesity effect of bioactive compounds in tea and coffee, Food Funct., 2016, 7, 4481-4491.

25 H. V. Sudeep and K. S. Prasad, Supplementation of green coffee bean extract in healthy overweight subject increases lean mass/fat mass ration: A randomized double-blind clinical study, SAGE Open Med., 2021, 9, 1-10.

26 F. Saura-Calixto, A. García-Alonso, I. Goñi and L. Bravo, In vitro determination of the indigestible fraction in foods: an alternative to dietary fiber analysis, J. Agric. Food Chem., 2000, 48, 3342-3347.

27 E. Lecumberri, R. Mateos, M. Izquierdo-Pulido, P. Ruperez, L. Goya and L. Bravo, Dietary fibre composition, antioxidant capacity and physico-chemical properties of a fibrerich product from cocoa (Theobroma cacao L.), Food Chem., 2007, 104, 948-954.

28 S. M. Tosh, Y. Brummer, S. S. Miller, A. Regand, C. Defelice, R. Duss, T. M. S. Wolever and P. J. Wood, Processing affects the physicochemical properties of $\beta$-glucan in oat bran cereal, J. Agric. Food Chem., 2010, 58, 7723-7730.

29 L. Bravo and F. Saura-Calixto, Characterization of dietary fiber and the in vitro indigestible fraction of grape pomace, Am. J. Enol. Vitic., 1998, 49, 135-141.

30 M. Gómez-Juaristi, S. Martínez-López, B. Sarria, L. Bravo and R. Mateos, Bioavailability of hydroxycinnamates in an instant green/roasted coffee blend in humans Identification of novel colonic metabolites, Food Funct., 2018, 9, 331-343.
31 J. Mataix-Verdú, L. García, M. Mañas-Almendros, E. Martínez-Victoria, J. Llopis-González, Tablas de Composición de Alimentos Españoles, Universidad de Granada, 4th edn, 2003.

32 S. Pal, A. Khossousi, C. Binns, S. Dhaliwal and V. Ellis, The effect of a fibre supplement compared to a healthy diet on body composition, lipids, glucose, insulin and other metabolic syndrome risk factors in overweight and obese individuals, Br. J. Nutr., 2011, 105, 90-100.

33 H. Yuan, P. Lan, Y. He, C. Li and X. Ma, Effect of the modifications on the physicochemical and biological properties of beta-glucan. A critical review, Molecules, 2019, 25, 57.

34 B. Du, M. Meenu, H. Liu and B. Xu, A concise review on the molecular structure and function relationship of $\beta$-glucan, Int. J. Mol. Sci., 2019, 20, 4032-4049.

35 T. A. Wilson, R. J. Nicolosi, B. Delaney, K. Chadwell, V. Moolchandani, T. Kotyla, S. Ponduru, G. H. Zheng, R. Hess, N. Knutson, L. Curry, L. Kolberg, M. Goulson and K. Ostergren, Reduced and high molecular weight barley beta-glucans decrease plasma total and non-HDL-cholesterol in hypercholesterolemic Syrian golden hamsters, J. Nutr., 2004, 134, 2617-2622.

36 T. M. S. Wolever, S. M. Tosh, A. L. Gibbs, J. Brand-Miller, A. M. Duncan, V. Hart, B. Lamarche, B. A. Thomson, R. Duss and P. J. Wood, Physicochemical properties of oat $\beta$-glucan influence its ability to reduce serum LDL cholesterol in humans: a randomized clinical trial, Am. J. Clin. Nutr., 2010, 92, 723-732.

37 A. Zurbau, J. C. Noronha, T. A. Khan, J. L. Sievenpiper and T. M. S. Wolever, The effect of oat beta-glucan on postprandial blood glucose and insulin responses: a systematic review and meta-analysis, Eur. J. Clin. Nutr., 2021, 75, 15401554.

38 K. N. Smith, K. M. Queenan, W. Thomas, R. G. Fulcher and J. L. Slavin, Physiological effects of concentrated barley beta-glucan in mildly hypercholesterolemic adults, J. Am. Coll. Nutr., 2008, 27, 434-440.

39 J. Frank, B. Sundberg, A. Kamal-Eldin, B. Vessby and P. Aman, Yeast-leavened oat breads with high or low molecular weight beta-glucan do not differ in their effects on blood concentrations of lipids, insulin, or glucose in humans, J. Nutr., 2004, 134, 1384-1388.

40 M. M-L. Grundy, A. Fardet, S. M. Tosh, G. T. Rich and P. J. Wilde, Processing of oat: the impact on oat's cholesterol lowering effect, Food Funct., 2018, 9, 1328-1343.

41 H. V. Ho, J. L. Sievenpiper, A. Zurbau, S. Blanco-Mejia, E. Jovanovski, F. Au-Yeung, A. L. Jenkins and V. Vuksan, A systematic review and meta-analysis of randomized controlled trials of the effect of barley $\beta$-glucan on LDL-C, nonHDL-C and apoB for cardiovascular disease risk reduction, Eur. J. Clin. Nutr., 2016, 70, 1239-1245.

42 E. F. Andrade, R. Vieira Lobato, T. Vasques de Araújo, M. G. Zangerônimo, R. V. de Sousa and L. J. Pereira, Effect of beta-glucans in the control of blood glucose levels of diabetic patients: a systematic review, Nutr. Hosp., 2015, 31, 170-177. 
43 F. Thies, L. F. Masson, P. Boffetta and P. Kris-Etherton, Oats and CVD risk markers: a systematic literature review, Br. J. Nutr., 2014, 112(Suppl 2), S19-S30.

44 H. R. Superko and R. R. Gadesam, Is it LDL particle size or number that correlates with risk for cardiovascular disease?, Curr. Atheroscler. Rep., 2008, 10, 377-385.

45 National Cholesterol Education Program (NCEP) Expert Panel on Detection, Evaluation, and Treatment of High Blood Cholesterol in Adults (Adult Treatment Panel III) final report, Circulation, 2002, 106, 3143-3421.

46 E. Jovanovski, R. Khayyat, A. Zurbau, A. Komishon, N. Mazhar and J. L. Sievenpiper, Should viscous fiber supplements be considered in diabetes control? Results from a systematic review and meta-analysis of randomized controlled trials, Diabetes Care, 2019, 42, 755-766.

47 X. L. Shen, T. Zhao, Y. Zhou, X. Shi, Y. Zou and G. Zhao, Effect of oat $\beta$-glucan intake on glycaemic control and insulin sensitivity of diabetic patients: a meta-analysis of randomized controlled trials, Nutrients, 2016, 8, 39.

48 C. J. Rebello, J. Burton, M. Heiman and F. L. Greenway, Gastrointestinal microbiome modulator improves tolerance in overweight and obese subjects: a randomized controlled pilot trial, J. Diabetes Complications, 2015, 29, 1272-1276.

49 E. Saltzman, S. K. Das and A. H. Lichtenstein, An oat-containing hypocaloric diet reduces systolic blood pressure and improves lipid profile beyond effects of weight loss in men and women, J. Nutr., 2001, 131, 1465-1470.

50 J. J. Pins, D. Geleva, J. M. Keenan, C. Frazel, P. J. O’Connor and L. M. Cherney, Do whole-grain oat cereals reduce the need for antihypertensive medications and improve blood pressure control?, J. Fam. Pract., 2002, 51, 353-359.

51 J. M. Keenan, J. J. Pins and C. Frazel, Oat ingestion reduces systolic and diastolic blood pressure in patients with mild or borderline hypertension: a pilot trial, J. Fam. Pract., 2002, 51, 369.

52 P. Tighe, G. Duthie and N. Vaughan, Effect of increased consumption of whole-grain foods on blood pressure and other cardiovascular risk markers in healthy middle-aged persons: a randomized controlled trial, Am. J. Clin. Nutr., 2010, 92, 733-740.
53 S. Aoe, K. Mio, C. Yamanaka and T. Kuge, Low molecular weight barley $\beta$-glucan affects glucose and lipid metabolism by prebiotic effects, Nutrients, 2021, 13, 130.

54 J. Rahmani, A. Miri, R. Černevičiūtè, J. Thompson, N. N. de Souza, R. Sultana and A. Hekmatdoost, Effects of cereal beta-glucan consumption on body weight, body mass index, waist circumference and total energy intake: A metaanalysis of randomized controlled trials, Complement. Ther. Med., 2019, 43, 131-139.

55 M. J. Clark and J. L. Slavin, The effect of fiber on satiety and food intake: a systematic review, J. Am. Coll. Nutr., 2013, 32, 200-211.

56 C. Berti, P. Riso, A. Brusamolino and M. Porrini, Effect on appetite control of minor cereal and pseudocereal products, Br. J. Nutr., 2005, 94, 850-858.

57 Y. Granfeldt, H. G. M. Liljeber, A. Drews, R. Newman and I. Björck, Glucose and insulin responses to barley products: influence of food structure and amylose-amylopectin ratio, Am. J. Clin. Nutr., 1994, 59, 1075-1082.

58 S. M. M. Zaremba, I. F. Gow, S. Drummond, J. T. McCluskey and R. E. Steinert, Effects of oat $\beta$-glucan consumption at breakfast on ad libitum eating, appetite, glycemia, insulinemia and GLP-1 concentrations in healthy subjects, Appetite, 2018, 128, 197-204.

59 T. M. S. Wolever, S. M. Tosh, S. E. Spruill, A. L. Jenkins, A. Ezatagha, R. Duss, J. Johnson, Y. Chu and R. E. Steinert, Increasing oat $\beta$-glucan viscosity in a breakfast meal slows gastric emptying and reduces glycemic and insulinemic responses but has no effect on appetite, food intake, or plasma ghrelin and PYY responses in healthy humans: a randomized, placebo-controlled, crossover trial, Am. J. Clin. Nutr., 2020, 111, 319-328.

60 L. Cloetens, M. Ulmius, A. Johansson-Persson, B. Åkesson and G. Önning, Role of dietary beta-glucans in the prevention of the metabolic syndrome, Nutr. Rev., 2012, 70, 444458.

61 B. Sarriá, S. Martínez-López, J. L. Sierra-Cinos, L. GarcíaDiz, R. Mateos and L. Bravo, Regularly consuming a green/ roasted coffee blend reduces the risk of metabolic syndrome, Eur. J. Nutr., 2018, 57, 269-278. 